\title{
TECHNOLOGY
}

\section{Transoesophageal echocardiography in the longitudinal axis: correlation between anatomy and images and its clinical implications}

\author{
O Stümper, A G Fraser, S Y Ho, R H Anderson, L Chow, M J Davies, J R T C Roelandt, \\ G R Sutherland
}

\begin{abstract}
Transoesophageal echocardiographic imaging in the longitudinal axis is a recent addition to the non-invasive evaluation of congenital and acquired heart disease. The technique provides unique images of intracardiac anatomy but their interpretation remains difficult. A heart specimen was therefore cut according to the echocardiographic imaging planes to elucidate the morphological details. The results suggested that longitudinal transoesophageal imaging complements the transverse axis approach. It gave new imaging information on the right ventricular outflow tract and the pulmonary trunk, the atrioventricular valves, the interventricular septum, the cardiac apex, and the thoracic aorta. In particular, it showed the entire length of the right ventricular outflow tract. When longitudinal imaging was used in combination with transverse imaging almost all the thoracic aorta could be examined. Imaging in the longitudinal axis may also allow better assessment of the mechanisms of atrioventricular valve regurgitation.
\end{abstract}

Thoraxcenter,

Erasmus University

Rotterdam,

The Netherlands

O Stümper

A G Fraser

J R T C Roelandt

G R Sutherland

National Heart and

Lung Institute,

University of London,

London

$S$ Y Ho

R H Anderson

St George's Hospital

Medical School,

London

L Chow

M J Davies

Correspondence to Dr G R Sutherland Department of Cardiology, Western General Hospital, Crewe Road, Edinburgh EH4 2XU.

Accepted for publication 24 April 1990
Transoesophageal echocardiography is evolving rapidly as an investigative and monitoring technique in the outpatient clinic and the operating theatre..$^{1-3}$ Studies are usually performed with a probe that consists of a single, phased array transducer mounted at the tip of an adapted gastroscope. This produces cross sectional images in planes at right angles to the shaft of the probe. Standard transoesophageal probes thus produce short axis scans through the heart and great vessels, most of which show cardiac morphology in anatomical transverse planes. ${ }^{45}$ This transoesophageal approach provides detailed insights into the systemic and pulmonary venous connections and into the morphology and function of the atria, the atrioventricular valves and each subvalvar apparatus, and the left ventricular outflow tract. ${ }^{6-8}$

Our experience of more than 1000 inves- tigations, including some 200 in patients with congenital cardiac malformations shows that scanning in a single plane in the transverse axis displays certain regions of the heart poorly. These include the right ventricular outflow tract and the apical and trabecular components of the interventricular septum. Furthermore, much of the ascending aorta is inaccessible to transoesophageal imaging in the transverse axis because the trachea is interposed between the aorta and the oesophagus.

The potential of scanning from within the oesophagus has recently been extended by the introduction of biplane, phased array probes by Omoto and his colleagues. ${ }^{9}$ These probes have a second transducer mounted at their tip that is oriented to scan in planes parallel to the shaft of the probe. Biplane transoesophageal probes therefore produce two cross sectional images in orthogonal planes, equivalent to transverse and longitudinal axis views of the heart. However, longitudinal axial scanning results in imaging planes that are unfamiliar.

To establish the anatomical basis for longitudinal transoesophageal echocardiographic imaging and to study its diagnostic potential we examined the morphological features of longitudinal anatomical sections of the heart and compared these with corresponding echocardiographic images obtained during clinical investigations.

\section{Patients and methods}

\section{TRANSOESOPHAGEAL ECHO STUDIES}

Transoesophageal imaging studies in both transverse and longitudinal planes were performed with a $5 \mathrm{MHz}$ biplane transoesophageal probe connected to an Aloka SSD 870 ultrasound system. Studies were carried out on outpatients and during cardiac surgery. The probe was rotated to obtain a complete series of longitudinal images between one hilum and the other, as well as the full range of transgastric views and views of the descending aorta. We describe the manipulation of the probe for an operator who is facing the patient. Flexion of the probe in both anterior/ posterior and lateral directions was used to optimise the image quality and increase the range of scan planes. 
Figure 1 Diagram of the heart showing the orientation of five of the standard sections that are obtained by longitudinal transoesophageal imaging.

Figure 2 ( $A$ )

Longitudinal axis scan

through the right atrium at

the site of drainage of the superior vena cava,

corresponding to section 1

in fig 1. The image is

displayed as it is seen on

the echocardiographic

monitor with the posterior aspect of the heart to the

top and the cranial aspect to the right. The vein is cut in its long axis; posteriorly the right pulmonary artery (RPA) is shown in short axis. The oval fossa of the interatrial septum is shown on the left of the scan; fibro-fatty tissue causes the bright echo between the atrial walls. (B) The corresponding anatomical section as seen from the right lateral aspect of the heart. It is displayed in a true anatomical

orientation, with the head to the top. $L A$, left

atrium; $R A$, right atrium; $S V C$, superior vena cava; $A$, anterior; $I$, inferior; $P$, posterior; $S$, superior.

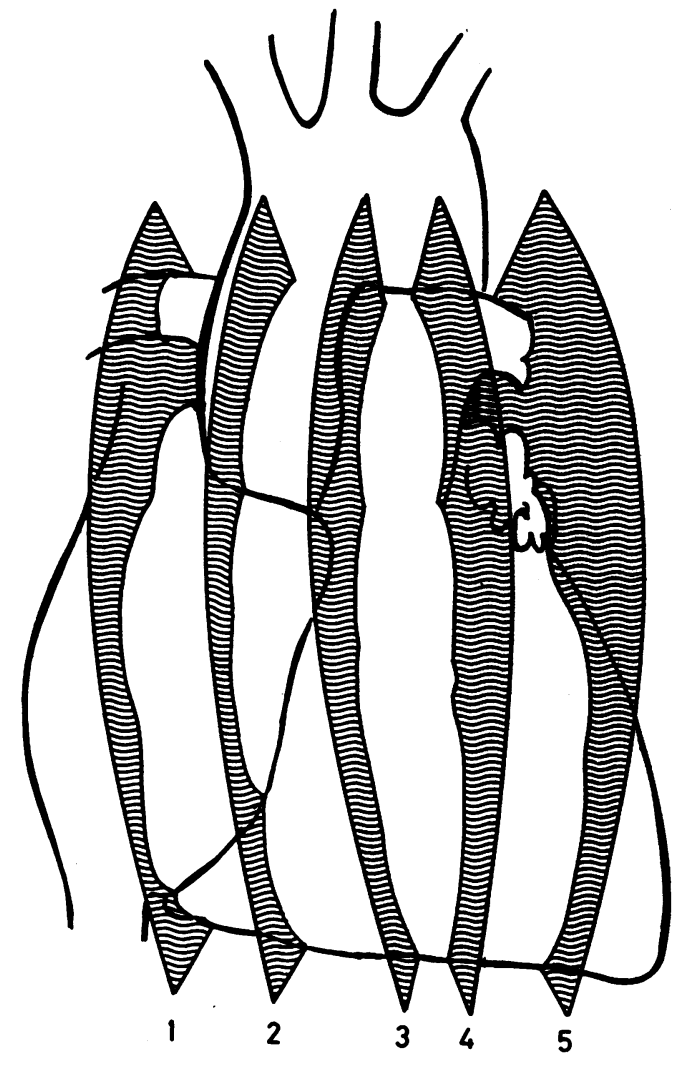

ANATOMICAL STUDY

A normal adult heart obtained at necropsy was perfused and fixed to preserve its shape. The proximal parts of the great vessels were retained, with the exception of the pulmonary arterial branches beyond the bifurcation. The specimen was first immersed in a waterbath (distilled water), and scanned in successive longitudinal planes from a point posterior to the left atrium, over the slight indentation caused by the oesophagus. These echocardiograms were performed with a standard precordial probe (short focus $5 \mathrm{MHz}$ transducer in a plastic sleeve containing ultrasound gel, connected to a Toshiba SSH-160A machine) and the images were recorded on videotape. Black and white prints were taken of relevant sections. The ultrasound studies were used to plan the site and orientation of corresponding anatomical sections of the heart, which were then prepared and photographed.

The anatomical sections are displayed in a true anatomical orientation, with the base of the heart and the great vessels at the top.

\section{Results}

Imaging the heart in its longitudinal axis produces many echocardiographic cross sections that are unique. The fulcrum from which

IMAGE ORIENTATION

The transoesophageal longitudinal axis scans, like the normal display of precordial images, are shown with the great arteries to the right side. The posterior aspect of the heart is displayed at the top of the image and the anterior aspect at the bottom, in keeping with the standard orientation of transoesophageal scans obtained in the transverse axis.
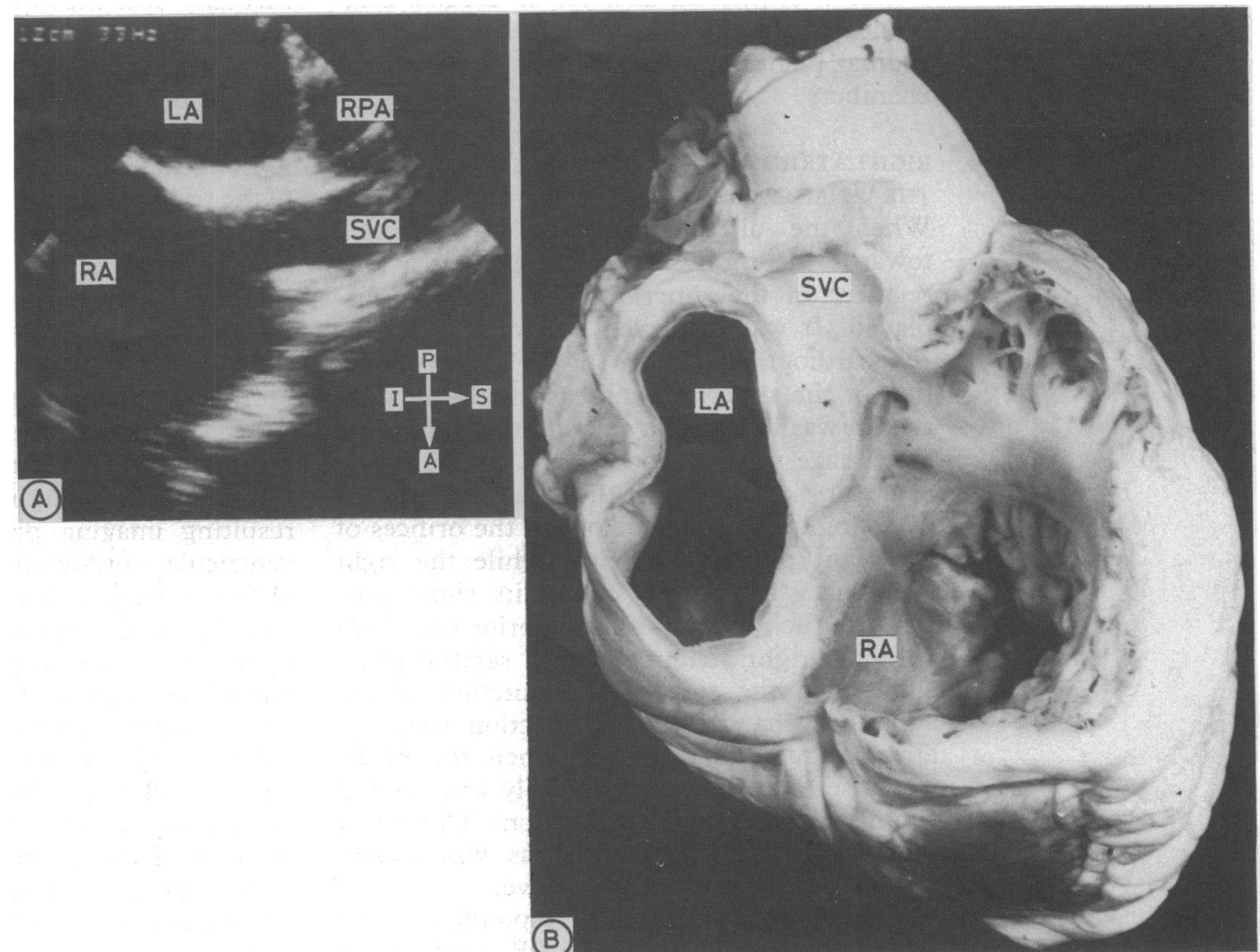
Figure 3 Longitudinal axis anatomical section corresponding to section 2 of fig 1 . The ascending aorta $(A o)$ is shown in its long axis and the orifice of the right coronary artery is shown. The section also cuts the anterosuperior and inferior leaflets of the tricuspid valve, and the distal coronary sinus. $L A$, left atrium; $R V$, right ventricle.

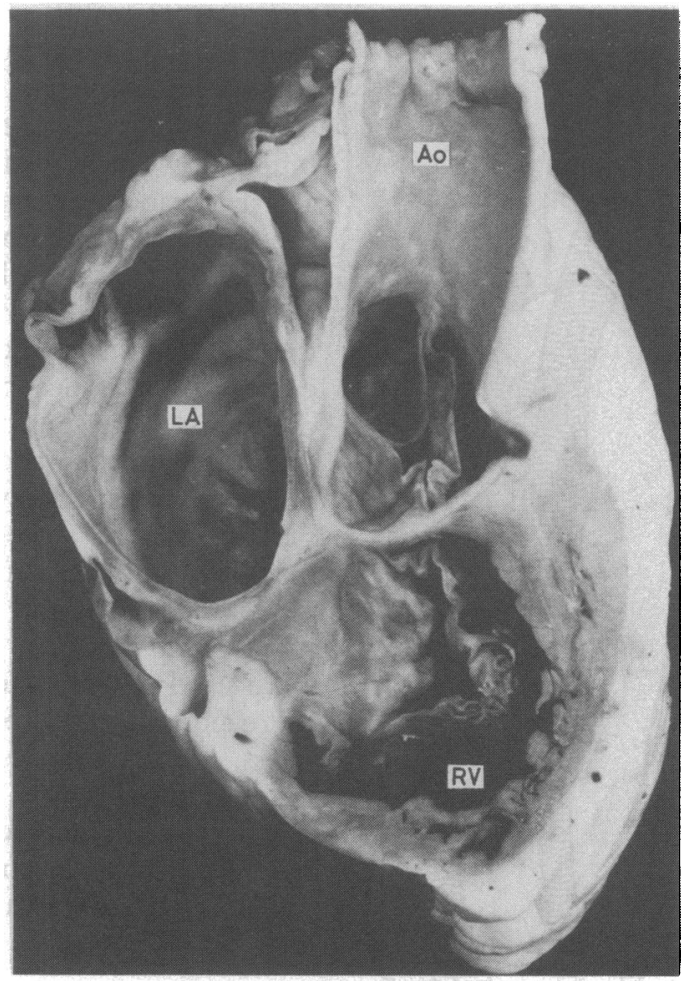

through smaller portions of the right heart and larger portions of the left heart. Together these scans show important features of the atria, the atrioventricular valves, the ventricles, and the arterial trunks. In addition, longitudinal imaging with the interrogating scan plane directed laterally towards either of the pulmonary hila can show the pulmonary veins and pulmonary arterial branches in cross section.

We present the transoesophageal images and their anatomical correlates according to the standard sequential analysis of cardiac morphology, although in practice most longitudinal planes traverse at least three cardiac chambers.

\section{RIGHT ATRIUM AND CONNECTIONS WITH THE VENAE CAVAE}

When the ultrasound beam was directed towards the right side of the patient a long segment of the superior vena cava was displayed. It was displayed horizontally on the echocardiographic image (fig 2A). The connection of the superior vena cava with the right atrium was well displayed, and the right atrial appendage was seen anteriorly, cut obliquely near its base. Posteriorly the left atrium was cut in a longitudinal plane close to the orifices of the right pulmonary veins, while the right pulmonary artery was cut in its short axis. Because the junction of the inferior vena cava with the right atrium lies in a sagittal plane slightly to the side of the junction of the superior vena cava, its connection with the atrium could be displayed when the probe was inserted a little more deeply and rotated slightly in a clockwise direction. The distal segment of the coronary sinus was shown within the atrioventricular groove.

The anatomical section corresponding to this echocardiographic plane (fig 2B) showed, in addition, that the interatrial septum was traversed medially and anteriorly to the thin oval fossa. Apparent thickening of the atrial septum around its edge was caused by fat between the free walls of the atria, that is in Waterston's groove. The true interatrial septum was a thin structure in the region of the oval fossa (fig $2 B$ ).

\section{TRICUSPID VALVE}

Slight anticlockwise rotation of the probe produced a more medial section through the right atrium and brought the tricuspid valve into view. The anterosuperior leaflet was displayed to the right of the image and the mural (or inferior) leaflet to the left. It was difficult with longitudinal imaging to define the commissures of the tricuspid valve. The zone of coaptation was also not well seen, because a considerable portion of it lay in almost a longitudinal plane and therefore the leaflets could not be scanned tangentially to the closure line. The lateral component of the right ventricular cavity was shown anterior to the tricuspid valve (fig 3 ).

Further anticlockwise rotation of the probe produced longitudinal scan planes that traversed the muscular portion of the atrioventricular septum, which lies between the offset attachments of the mitral and tricuspid valve leaflets. Imaging of the smaller membranous portion of the atrioventricular septum was difficult because it lay in almost a longitudinal plane and was thus crossed at a very shallow angle.

Depending on the relative sizes and anatomical relation of the ascending aorta and the superior vena cava, longitudinal sections through the vein sometimes included an elliptical section of the right side of the anteriorly positioned ascending aorta. In all patients, the aorta was seen as the scan plane was shifted medially and the aortic valve was sectioned. The first cusps to be displayed were those within the right and non-coronary sinuses (fig 3). The orifice of the right coronary artery was also shown.

Longitudinal scans through the medial part of the tricuspid valve also brought the orifice of the coronary sinus into view, and the transverse sinus of the pericardial cavity was well seen between the posterior aspect of the aorta and the anterior wall of the left atrium.

\section{RIGHT VENTRICULAR OUTFLOW TRACT}

When the longitudinal axis transducer was positioned so that it scanned directly forward from the oesophagus in a true sagittal plane, the resulting imaging plane traversed the right ventricular outflow tract tangentially. If the tip of the probe was then angled to the right (fig 4A) the whole length of the right ventricular outflow tract was displayed together with the pulmonary valve, the proximal pulmonary trunk, and the anterolateral free wall of the right ventricle in the same view (fig 4B). The septal leaflet of the tricuspid valve was sometimes included in this section, and the atrioventricular portion of the membranous septum was crossed superiorly. The aortic root was located centrally in this view and all three cusps were sectioned (fig 4C). 
Figure 4 (A) Diagram showing the scan plane that displays the right

ventricular outflow tract. This is obtained by tilting the transducer laterally from position 3 in fig 1 .

(B) Longitudinal axis scan through the right ventricular cavity. ( $R V)$, the right ventricular outflow tract, and the proximal pulmonary trunk (PT). Centrally the aortic root $(A o)$ is cut obliquely. Postero-

inferiorly, a segment of the right atrium $(R A)$ is seen together with a portion of the atrioventricular junction and the anterosuperior leaflet of the tricuspid valve. (C) $A$ corresponding anatomical section. $L A$, left atrium. See fig 2 legend for other abbreviations.

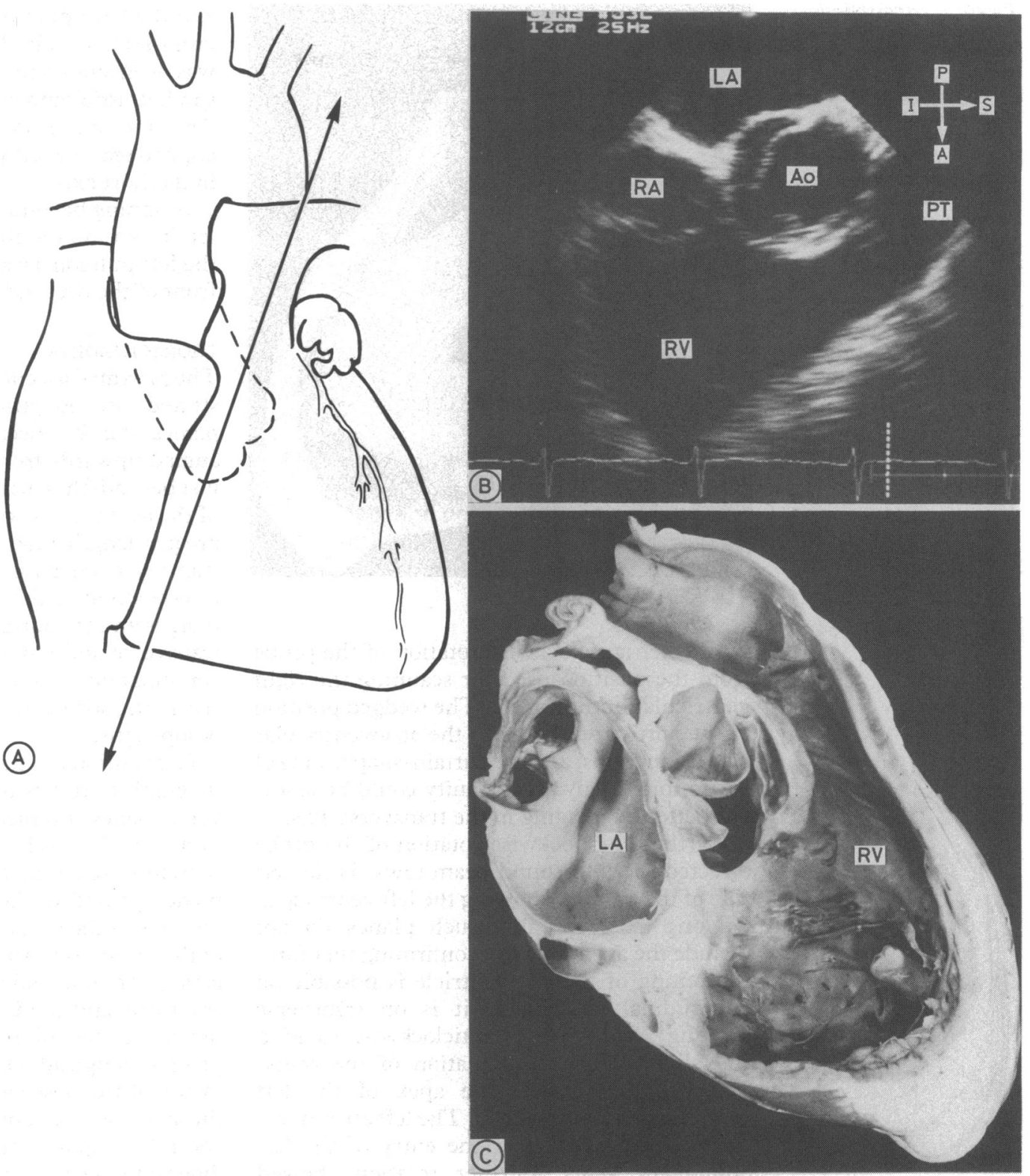

Slight clockwise or counter-clockwise rotation of the probe from the central position used to scan the right ventricular outflow tract showed the pulmonary arterial branches near the bifurcation and these could be followed in cross section towards the hila. The right pulmonary artery was seen and displayed behind the ascending aorta, while the left pulmonary artery was seen above and in front of the left atrial appendage.

\section{LEFT ATRIUM, MITRAL VALVE, AND LEFT VENTRICLE}

When the probe was rotated in an anticlockwise direction from the longitudinal scans of the right heart towards the left, the left ventricle was first shown near the posteromedial commissure of the mitral valve, with the muscular portion of the ventricular septum crossed tangentially (fig 5). The apical part of the right ventricle was shown as a rim anteriorly and the muscular septum was shown in oblique section. A small portion of the interventricular component of the membranous septum was still visualised cranially.
Continued anticlockwise rotation could be used to study the coaptation of the leaflets of the mitral valve in longitudinal planes. Between the posteromedial commissure and the middle of the leaflets, these planes crossed the zone of coaptation approximately at right angles to the apposed edges of the leaflets (fig 6). More laterally, the imaging planes became more or less parallel to the zone of coaptation as it curved upwards towards the left atrial appendage. Thus with longitudinal imaging the relations between the leaflets over their lateral thirds and near the anterolateral commissure are difficult to ascertain. Indeed, the space between the middle and lateral scallops of the mural (posterior) leaflet could be confused with the zone of coaptation. Because the papillary muscles lie almost in the same longitudinal plane, they may be seen together on one echocardiographic image.

The left ventricular outflow tract was cut obliquely when it was assessed in a series of longitudinal scans. Continuity between the leaflets of the aortic and mitral valves and the left coronary sinus of the aorta could be shown 
Figure 5 Anatomical section through the muscular interventricular septum (IVS) corresponding to section 4 in fig 1 . The coaptation zone of the mitral valve is crossed near the posteromedial commissure. The section also shows the left coronary cusp and part of the adjacent sinus of Valsalva. $L A$, left atrium, $P T$, pulmonary trunk.

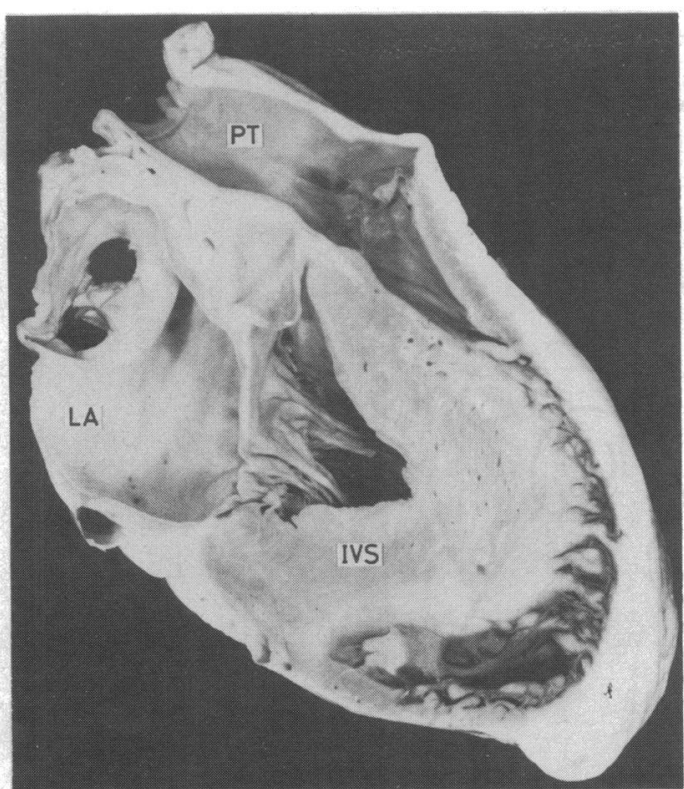

by further anticlockwise rotation of the probe from the position used for scanning the right ventricular outflow tract. The wedged position of the aortic root between the atrioventricular valvar junctions, and the curtain-shaped area of aortic-mitral valvar continuity could be assessed better by imaging in the transverse axis.

Further anticlockwise rotation of the probe directed the ultrasound beam towards'the left side of the patient, showing the left ventricle in its long axis. Initially, such planes do not include the apex, thereby confirming that foreshortening of the left ventricle is possible on longitudinal imaging as it is on transverse imaging. With further anticlockwise rotation and slight leftward angulation of the transducer, however, the true apex of the left ventricle was shown (fig 7). The left atrium was traversed laterally, near the entry of the left pulmonary veins. Further rotation showed both the left atrial appendage in its long axis

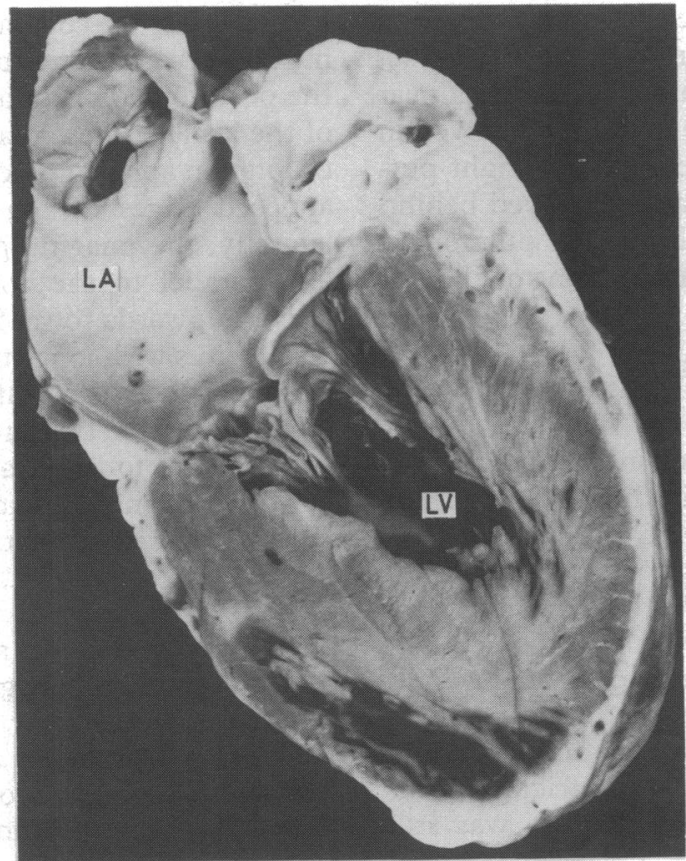

and the left upper pulmonary vein in the same cross section. The left upper pulmonary vein was seen cranioposteriorly to the junction of the left atrial appendage with the left atrium. Anterior and also cranial to the left atrial appendage, the left pulmonary artery was seen in its short axis.

Scanning beyond the left atrium towards the left hilum showed the left pulmonary veins and the left pulmonary artery in their short axes in front of the descending aorta (fig 8).

\section{THORACIC AORTA}

The proximal ascending aorta was well demonstrated in longitudinal echocardiographic planes (fig 9). Because the probe could be angled upwards from a site in the oesophagus just beneath the carina, the mid and upper parts of the ascending aorta could be imaged along a greater length than is possible with standard transverse imaging, which is limited by the interposition of the trachea between the oesophagus and the aorta. Nevertheless, even when transverse and longitudinal axes were used in combination, a short segment of the upper ascending aorta remained inaccessible from the oesophagus.

Conventional transoesophageal echocardiographic studies of the aortic arch in transverse planes produce long ellipsoid cross sections of the arch. As would be expected, therefore, scanning of the arch in longitudinal planes provided short axis or circular cross sections of the aorta (fig 10). A segment of one of the pulmonary arteries adjacent to the bifurcation of the pulmonary trunk was seen anteriorly and caudally. With the probe directed to the left or posteriorly from the oesophagus, longitudinal scans showed long segments of the descending aorta (fig 8), making the assessment of abnormalities of the thoracic aorta by biplane transoesophageal echocardiography faster and more detailed than is possible with single plane imaging.

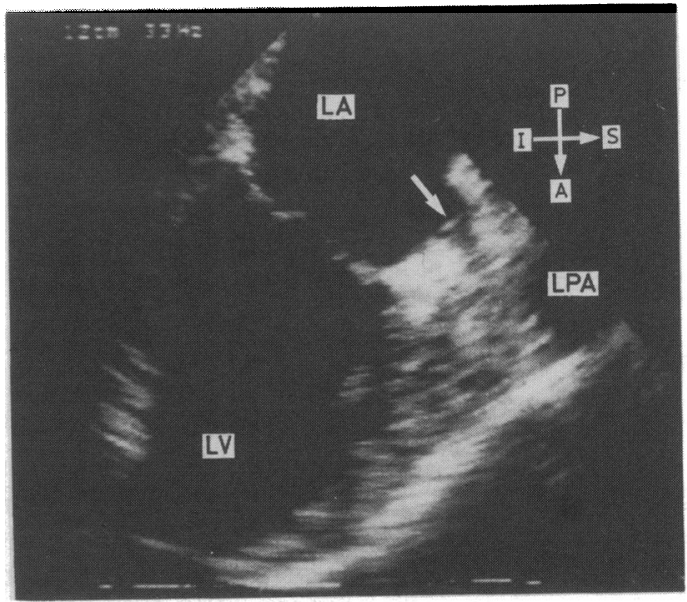

Figure 7 Longitudinal axis scan showing the left ventricle ( $L V$ ) in its long axis and including the left ventricular apex. This view was obtained with slight left lateral angulation of the transducer. The zone of coaptation between the anterior (aortic) and posterior (mural) leaflets of the mitral valve is shown as is the junction (arrow) of the left atrial appendage with the left atrium $(L A)$. The left pulmonary artery (LPA) is cut in its short axis. 


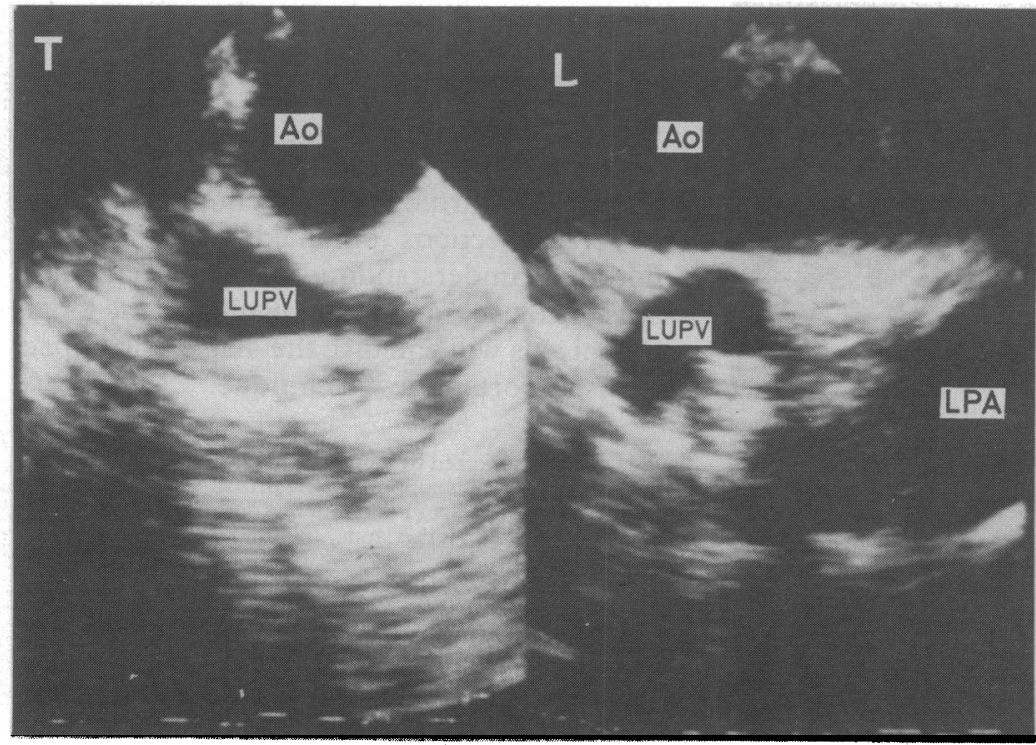

Figure 8 Transverse $(T)$ and longitudinal $(L)$ axial scans of the descending aorta (Ao) at the level of the left upper pulmonary vein (LUPV). Long segments of the anterior and posterior walls of the descending aorta are shown on the same longitudinal scan. Anteriorly the left pulmonary artery (LPA) and the left upper pulmonary vein are cut in short axis at their peripheral branching. longitudinal imaging planes. This study and our initial clinical experience suggest that the technique will be a valuable addition to standard transoesophageal imaging in the transverse axis in the assessment of specific aspects of both congenital and acquired heart disease.

In congenital heart disease, imaging in the longitudinal axis showed the entire length of the right ventricular outflow tract; this was almost impossible when transverse axial planes were used on their own. In addition, the technique may enhance the assessment of overriding of the arterial valves over a ventricular septal defect. The planes were useful in determining the integrity of the interventricular septum and in showing the deviation and shape of its components and the relation of the ventricular chambers one to the other. Imaging in the longitudinal axis, therefore, may be expected to have considerable clinical impact on the echocardiographic assessment of complex congenital heart disease in adolescents and in adults with poor precordial windows.

In patients with acquired heart disease, early experience suggested that the technique will be most useful in assessing disease of the thoracic aorta, ${ }^{17}$ such as dissection or aneurysm. The combination of imaging in the longitudinal and transverse planes now allows an almost complete echocardiographic assessment of the aorta between the aortic valve and the diaphragm. This should prove invaluable in the urgent diagnosis of patients with acute chest pain and suspected aortic dissection. However, even biplane transoesophageal imaging cannot display a small portion of the upper ascending aorta and the proximal arch, although this is considerably smaller than the area that is inaccessible to imaging in the transverse plane alone. Finally, colour flow mapping in the longitudinal axis will contribute towards a better understanding of the nature and shape of lesions of the aorta. For example, it may allow more rapid and accurate distinction between true and false lumens in aortic dissection.

Imaging in the longitudinal axis may also be of value in patients with diseases of the aortic root, such as infective endocarditis, and in those with prosthetic valve dysfunction, because it allows the examination of additional imaging planes. Although the technique cannot on its own provide a complete analysis of atrioventricular valvar shape, longitudinal imaging displays the anterosuperior and mural (inferior) leaflets of the tricuspid valve and it is useful for analysing coaptation over the medial half of the mitral valve. Thus it can complement the information that is obtained by precordial and transverse transoesophageal techniques. Biplane transoesophageal echocardiography may be used to obtain colour flow maps of regurgitant jets that can be analysed in orthogonal planes.

Scanning in the longitudinal axis from within the oesophagus has some limitations compared with transverse axial imaging. For example, it did not improve visualisation of the branches of the pulmonary trunk, which are always displayed either in oblique or short axes. In particular, no additional information is image) a small segment of the proximal pulmonary trunk (PT) are also shown. The tricuspid valve and the right ventricle are not shown on this high longitudinal image, which corresponds to the upper half of the anatomical section in fig 3. 


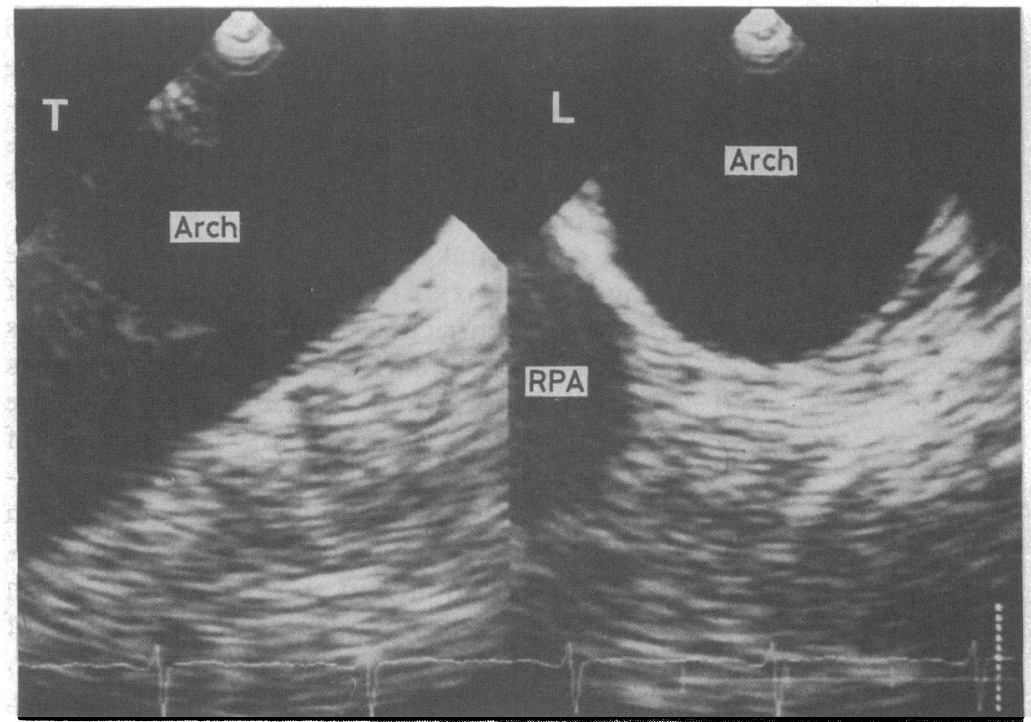

Figure 10 Transverse $(T)$ and longitudinal $(L)$ axis scans of the aortic arch. The aortic arch (Arch) is shown almost in its long axis on the transverse axis scan, whereas it is cut in short axis on the longitudinal scan. A segment of the proximal right pulmonary artery $(R P A)$ is displayed inferiorly. when prior clinical and echocardiographic assessment suggests that it may provide diagnostic images of unique value.

The addition of imaging in the longitudinal axis complements the standard transoesophageal echocardiographic technique. Some of the cross sections obtained are unique. A thorough understanding of the anatomical basis of transoesophageal imaging in the longitudinal axis will enhance the diagnostic impact of this important new technique.

OS is in part supported by a grant of the Deutsche Forschungsgemeinschaft; AGF is supported by a British-Dutch Fellowship of the British Heart Foundation. We thank Aloka/IMS Biomedic bv, The Netherlands, for their assistance with this
project.

1 Schlüter M, Hanrath P. Transesophageal echocardiography: potential advantages and initial clinical results. phy: potential advantages and in
Practical Cardiology 1983;9:149-80.

2 Gussenhoven EJ, Taams MA, Roelandt JRTC, et al. Transesophageal two dimensional echocardiography: its role in solving clinical problems. J Am Coll Cardiol role in solving
$1986 ; 8: 975-79$.

3 Abel MD, Nishimura RA, Callahan MJ, Rehder K, Ilstrup DM, Tajik AJ. Evaluation of intraoperative transoesophageal two-dimensional echocardiography. Anesthesiology 1987;66:64-8.

obtained on the course and shape of the left pulmonary artery, and transoesophageal imaging of this vessel remains difficult.

The longitudinal axial approach cannot contribute significantly to the haemodynamic evaluation of either congenital or acquired heart disease. The assessment of flow across the atrioventricular valves is similar with both approaches. Although the pulmonary trunk and the right ventricular outflow tract are much better displayed by longitudinal than by transverse imaging, pulsed Doppler interrogation in longitudinal planes is less rewarding, because the angle of incidence between the ultrasonic beam and the flow of blood in these structures always approaches $90^{\circ}$. Therefore, even when both planes are used in combination, the haemodynamic evaluation of cardiac lesions will require precordial ultrasound.

A final technical consideration that militates against the use of biplane transoesophageal probes for all routine transoesophageal studies is that the number of elements in each transducer is less than what is normally available with transducers designed for use in a single plane. Indeed, the high definition of standard transoesophageal imaging is a major advantage of the technique. At present, however, there is an inevitable compromise in biplane imaging because of the need to incorporate the electronic connections for two transducers within the shaft of one probe. These considerations account for the definition of the echocardiographic images presented in the present report which is less than what is now considered routine from single plane transoesophageal studies. In our opinion, biplane transoesophageal imaging should be performed only 